\title{
ITPA Index in neurosyphilis laboratory diagnosis.
}

\author{
Negasheva Ekaterina*, Dmitriev Georgiy, Potekaev Nikolay, Frigo Nataliia \\ Moscow Scientific and Practical Center of Dermatovenereology and Cosmetology DZM, Moscow, Russia
}

\begin{abstract}
Neurosyphilis (NS) is an interdisciplinary problem, in which a whole range of specialists should participate: dermatovenereologists, laboratory assistants, neuropathologists, psychiatrists, ophthalmologists, and others, depending on the disease manifestations. In order to verify the diagnosis and differentiate it from lesions of the nervous system associated with other causes, instrumental methods of examining patients should be widely used. Using instrumental methodologies is limited and the symptoms of the disease in many cases are nonspecific. NS diagnosis is based on laboratory diagnostics. In this regard, in recent years, numerous studies aimed at optimizing the clinical and laboratory diagnosis of this disease. For several years, a group of specialists carried out comparative clinical and laboratory studies, the development of immunochips for neurosyphilis diagnosis, and an assessment of the diagnostic significance of the ITPA index (serum-liquor ratio). Having analysed the most interesting scientific and applied developments of foreign and Russian colleagues, as well as the results of our own research, we came to the conclusion that the most promising, from a practical point of view, is the serum-liquor ratio in paired samples of patients with suspicion of neurosyphilis and, possibly, other forms of the disease. It seems that, with the help of this methodology, it is possible to obtain reliable data on the presence (absence) of central-nervous-system pathology in syphilis, and, after obtaining additional results, and on the effectiveness of therapeutic effects on the normalization (tendency to decrease the ITPA index) of cerebrospinal fluid.
\end{abstract}

Keywords: Neurosyphilis, CSF, Laboratory diagnosis, Syphilis.

Accepted on December 26, 2018

\section{Introduction}

Neurosyphilis (NS) is an interdisciplinary problem, in which a whole range of specialists should participate: dermatovenereologists, laboratory assistants, neuropathologists, psychiatrists, ophthalmologists, and others, depending on the disease manifestations. In order to verify the diagnosis and differentiate it from lesions of the nervous system associated with other causes, instrumental methods of examining patients should be widely used [1].

The current international classification does not fully reflect the clinical forms of the disease; the diagnosis of neurosyphilis without neurological symptoms is casuistic, especially in cases of absence of the pathology of the cerebrospinal fluid (CSF); the same applies to unspecified NS.

The mechanism of NS formation is largely unclear. At the same time, it is considered proven that, in most cases, passive Treponema pallidum transport and its compartments through CSF or spinal fluid is noted without the formation of pathology of the nervous system, which is apparently associated with a blood-brain barrier violation.

The use of instrumental methodologies is limited, and the symptoms of the disease in many cases are nonspecific. In establishing an NS diagnosis, it is necessary to base it on laboratory diagnostics, which is the most objective component of an integrated approach to managing patients. In this regard, in recent years, numerous studies aimed at optimizing the clinical and laboratory diagnosis of this disease $[2,3]$.

\section{Procedure}

For several years, a group of specialists carried out comparative clinical and laboratory studies, the development of immunochips for neurosyphilis diagnosis, and an assessment of the diagnostic significance of the ITPA index (serum-liquor ratio).

Conducting large-scale studies, we paid attention to the fact that a significant number and, in some cases, the vast majority of patients with NS, had no CSF pathology: negative results of nontreponemal tests were recorded, and pleocytosis and hyperproteinarchy (low protein content and cell count) were not observed. In other words, the Merritt triad postulate [4], which considered the presence of the above-mentioned positive indicators to be sufficient basis for making an NS diagnosis, was not applicable in this case.

A new class of reagents for the laboratory diagnosis of infectious diseases has been created: immunochips, the principle of which is based on an indirect method of detecting 
antibodies in the antigen spectrum (fluorescence or phosphorescence). A diagnostic test system consists of an immunosorbent with $T$. pallidum antigens that are separately immobilized on it, Tp15, Tp17, Tp47, and TmpA, which allows the simultaneous and differentiated detection of reagin and treponemospecific antibodies in pale treponem. This methodology was clinically tested to identify antibodies to $T$. pallidum in the CSF and to verify the diagnosis of neurosyphilis. As a result of the research, its high efficiency was established: the diagnostic sensitivity and specificity of the immunochip when testing CSF samples obtained from patients with an established diagnosis of neurosyphilis and from patients without it were $100 \%$ and $100 \%$, respectively.

Thus, convincing evidence was obtained that an immunochip using four recombinant pale T. pallidum antigens (Tp15, Tp17, Tp47, and TmpA) could be successfully applied in clinical practice. The methodology of determining antibodies for $T$. pallidum in an immunochip format improves the accuracy of NS diagnosis and allows the simultaneous examination of a significant number of patients. The possibility of its use in screening and confirmatory tests allows reducing the cost of laboratory tests for syphilis and neurosyphilis in the future.

It should be noted that, in other forms and stages of syphilis, obtaining positive results from specific (treponemal) and nonspecific (nontreponemal) tests is a significant basis (along with clinical data) for diagnosis, but in neurosyphilis this is not enough. The basis of laboratory diagnosis of NS is the establishment of the intrathecal (intracanal) synthesis of treponemal antibodies, and its differentiation from passive transfusion transfer of specific antibodies through a damaged blood-brain barrier (BBB) from the blood to the cerebrospinal fluid. Thus, the basis of a fundamentally new scientific and applied approach to the diagnosis of neurosyphilis and, possibly, other forms of syphilis, may be the study of the serum-liquor ratio $[5,6]$.

In this regard, we carried out a practical study of the methodology for determining the intrathecal synthesis of antitreponemal immunoglobulins by determining the serumliquor ratio-the so-called ITPA index.

The concept of this methodology is that in normal (without central-nervous-system (CNS) pathology) systems, the ratio of specific IgG in the cerebrospinal fluid and serum is identical (equal to 1), i.e., if antibodies to $T$. pallidum are not synthesized in the central nervous system, the presence of treponemospecific antibodies in the CSF is a result of their passive transfer from the blood through the BBB. If the value of the ITPA index is equal to two or more, the possibility of the formation of NA should be assumed; in this case, the appointment of preventive (prophylactic) therapy can stop this process. An index value of three or more (three to 430) clearly indicates intrathecal synthesis of specific antibodies and, accordingly, specific lesion of the nervous system caused by a syphilitic infection.

In view of the above, we carried out comparative studies of paired bioassays obtained from 65 patients in standard tests and the ITPA-index methodology. Result analysis demonstrated the absence of pleocytosis and hyperproteinarchy in the cerebrospinal fluid, as well as negative RMP values and a low percentage of treponema immobilization in RITs of no more than 68. Other specific treponemal tests (RPHA, ELISA, RIF) with NS were mostly positive. The most demonstrative were the data obtained by the methods of RPGA, RIF, and ELISA (97.7\%; 97.7\%, and 94\% positive results, respectively).

Positive results of treponemal and nontreponemal tests were found in the blood serum of the same patients.

Thus, the results obtained using known methodologies could not confirm or refute NS diagnosis, and positive treponemal tests in serum testified only in favor of syphilitic infection without nervous-system pathology.

Studies of paired bioassays of these same patients using the methodology for determining the serum-liquor ratio showed the following. In the majority of patients with clinically established NS diagnoses, the ITPA index ranged from 9.06 to 260.8, which corresponded to the presence of neurosyphilis. The exceptions were the samples of two patients with ANS, the index values of which amounted to 1.88 , which corresponded to the "borderline" state. However, the presence of clinical and other laboratory signs of NS (RPGA, RIFTS) and obtaining repositive values of the ITPA index made it possible to confirm the NS diagnosis. Thus, NS diagnosis using the ITPA index was established in all $(100 \%)$ patients.

It was noteworthy that, in the group of patients with NS with symptoms, the ITPA index was significantly higher than the group with NS without symptoms. However, the reliability of this phenomenon is to be proved on more significant factual material.

In the vast majority of patients in the comparison group, neurosyphilis was not detected according to the ITPA index. In two bioassays, the index values were 1.8-1.9, which corresponded to an uncertain result; re-examining the bioassay showed a negative result. In biological samples of two patients (9.0\%), ITPA-index values were 3.6-4.38, which indicated the formation of CNS pathology and required changes in patientmanagement tactics and the appointment of adequate specific therapy.

Thus, the ITPA index was a highly informative indicator in NS diagnosis. The difference in absolute values of the index between patients with NS and syphilis without NS was highly reliable $(\mathrm{p}<0.001)[7,8]$.

A relatively small number of bioassay studies obtained from patients with neurosyphilis and other forms of the disease do not currently provide an opportunity for an unambiguous interpretation of the results obtained using this methodology. However, if the ITPA index is sufficiently high as a "cure criterion" (during and after therapeutic interventions), this methodology could be among the regulated tests for verifying the laboratory diagnosis of various forms of syphilis, especially since it does not require separate regulation, additional equipment, and highly qualified personnel, and it simplifies 
and shortens the terms of the formulation, as well as the economic costs in the examination and management of patients.

\section{Conclusion}

After analyzing the most interesting, in our opinion, scientific and applied developments, as well as the results of our own research, we came to the conclusion that the most promising development, from a practical point of view, is the study of the serum-liquor ratio in paired samples of patients with suspected neurosyphilis and possibly other forms of the disease. It seems that, by using this methodology, one can obtain reliable data on the presence (absence) of central-nervous-system pathology in syphilis as well as, after obtaining additional results, on the effectiveness of therapeutic effects on the normalization (ITPA-index decrease tendency) of cerebrospinal fluid.

\section{Funding}

This research received no external funding.

\section{References}

1. Bollensen E, Albrecht S, Beuche W, Mader M, Prange HW. Reactivity of locally produced CSF antibodies in patients with neurosyphilis against antigens of Treponema pallidum. J Neurol 1993; 240: 471-474.

2. Castro R, Prieto ES, da Luz Martins Pereira F. Nontreponemal tests in the diagnosis of neurosyphilis: an evaluation of the venereal disease research laboratory (VDRL) and the rapid plasma reagin (RPR) tests. J Clin Lab Anal 2008; 22: 257-614.
Larsen SA, Hambie EA, Wobig GH, Kennedy EJ. Cerebrospinal fluid serologic test for syphilis: Treponemal and nontreponemal tests. VNW Science Press Utrecht, Netherlands 1985; 157.

3. Merrit H, Adams R, Solomon H. Neurosyphilis. Oxford University Press: New York, NY, USA 1946; 430.

4. Prange HW, Moskophidis M, Schipper HI, Muller F. Relationship between neurological features and intrathecal synthesis of IgG antibodies to Treponema pallidum in untreated and treated human neurosyphilis. J Neurol 1983; 230: 241-252.

5. Muller F, Moskophidis M. Estimation of the local production of antibodies to Treponema pallidum in the central nervous system of patients with neurosyphilis. Br J Vener Dis 1983; 59: 80-84.

6. Dmitriev GA. The role of serum-liquor ratio in the diagnosis of neurosyphilis. Wedge Dermatol Venereol 2015; 14: 40-45.

7. Dmitriev GA, Potekaev NN, Negasheva ES, Frigo NV. ITPA-index in the clinical and laboratory diagnosis of neurosyphilis. Wedge Dermatol Venereol 2017; 16: 38-43.

\section{*Correspondence to}

Negasheva Ekaterina

Moscow Scientific and Practical Center for Dermatology and Venereology and Cosmetology

Moscow

Russia 